\title{
A NOTE ON LIFTINGS OF HERMITIAN ELEMENTS AND UNITARIES
}

\author{
by C. K. FONG
}

(Received 25 May, 1979)

Let $A$ be a complex Banach algebra with unit 1 satisfying $\|1\|=1$. An element $u$ in $A$ is said to be unitary if it is invertible and $\|u\|=\left\|u^{-1}\right\|=1$. An element $h$ in $A$ is said to be hermitian if $\|\exp (i t h)\|=1$ for all real $t$; that is, $\exp (i t h)$ is unitary for all real $t$. Suppose that $J$ is a closed two-sided ideal and $\pi: A \rightarrow A / J$ is the quotient mapping. It is easy to see that if $x$ in $A$ is hermitian (resp. unitary), then so is $\pi(x)$ in $A / J$. We consider the following general question which is the converse of the above statement: given a hermitian (resp. unitary) element $y$ in $A / J$, can we find a hermitian (resp. unitary) element $x$ in $A$ such that $\pi(x)=y$ ? (The author has learned that this question, in a more restrictive form, was raised by F. F. Bonsall and that some special cases were investigated; see [1], [2].) In the present note, we give a partial answer to this question under the assumption that $A$ is finite dimensional.

For notation and terminology, we follow the book by Bonsall and Duncan [3]. We shall always assume that $A$ is finite dimensional.

Note that there exists an idempotent $e$ in $A$ which is minimal with respect to the property that $\|e\|=1$ and $1-e \in J$; that is, if $f$ is an idempotent such that $e f=f e=f,\|f\|=1$ and $1-f \in J$, then $e=f$. Also note that $e A e$ is a Banach algebra with $e$ as its unit, $e A e+J=A$ and an element $h$ in $e A e$ is a hermitian element in the algebra $e A e$ if and only if $\|\exp (i t h) e\|=1$ for all real $t$.

THEOREM A. If $h$ is a hermitian element in $A / J$, then there is a hermitian element $\bar{h}$ in eAe such that $\pi(\tilde{h})=h$.

THEOREM B. If $u$ is a unitary element in $A / J$, then there exists a unitary element $\tilde{u}$ in eAe such that $\pi(\tilde{u})=u$.

To prove these theorems, we need some technical lemmas. First we note that, since $A$ is finite dimensional, for $x \in A$, the spectrum $\mathrm{Sp}(x)$ is a finite set. For $\lambda \in \mathrm{Sp}(x)$, we shall write $e(\lambda, x)$, or simply $e_{\lambda}$ if this does not cause confusion, for the idempotent

$$
\frac{1}{2 \pi i} \int_{\partial D_{\lambda}}(\zeta-x)^{-1} d \zeta
$$

where $D_{\lambda}$ is a closed disc with $\lambda$ as its center and $D_{\lambda} \cap \operatorname{Sp}(x)=\{\lambda\}$.

LemmA 1. If $x \in A$ and $\lambda \in \operatorname{Sp}(x) \backslash \operatorname{Sp}(\pi(x))$, then $e_{\lambda} \in J$.

Proof. Since $\lambda \notin \operatorname{Sp}(\pi(x))$, we have

$$
\pi\left(e_{\lambda}\right)=\frac{1}{2 \pi i} \int_{\partial D_{\lambda}}(\zeta-\pi(x))^{-1} d \zeta=e(\lambda, \pi(x))=0 .
$$

Therefore $e_{\lambda} \in J$.

Glasgow Math. J. 21 (1980) 183-185. 
Lemma 2. If $x \in A,\|x\|=1, \lambda \in \mathrm{Sp}(x)$ and $|\lambda|=1$, then $e_{\lambda} x=x e_{\lambda}=\lambda e_{\lambda}$ and $\left\|e_{\lambda}\right\|=1$.

Proof. Consider the left regular representation $\Lambda: A \rightarrow B L(A)$ defined by $\Lambda(a) z=$ az. Let $T=\Lambda(x)$. Then $\operatorname{Sp}(T)=\operatorname{Sp}(x)$. Let $P_{\lambda}$ be the spectral projection $e(\lambda, T)$. Then it is easy to show that $\Lambda\left(e_{\lambda}\right)=P_{\lambda}$. Since $\|T\|=1$ and $|\lambda|=1$, it follows from [4] that the range of $P_{\lambda}$ is the eigenspace $\{w \in A: T w=\lambda w\}$ and $\left\|P_{\lambda}\right\| \leq 1$. Hence $\left\|e_{\lambda}\right\|=1$. From $T P_{\lambda}=\lambda P_{\lambda}$ and the fact that $\Lambda$ is one-one, we have $x e_{\lambda}=\lambda e_{\lambda}$.

Proof of Theorem $A$. For each real number $t$, let

$$
K_{t}=\{x \in e A e:\|x\|=1 \text { and } \pi(x)=\exp (i t h)\} .
$$

Note that $K_{t}$ is non-empty for each real $t$. (In fact, let $y \in A$ be such that $\pi(y)=\exp (i t h)$. Then

$$
1=\|\exp (i t h)\|=\inf \{\|y+z\|: z \in J\} .
$$

By a compactness argument, we see that there exists some $z \in J$ such that $\|y+z\|=1$. Now one can check that $e(y+z) e \in K_{t}$.) It is straightforward to verify that $K_{s} K_{t} \subseteq K_{s+t}$ and that the graph of the set-valued mapping $t \rightarrow K_{t}$ given by

$$
\left\{(t, x) \in \mathbf{R} \times A: x \in K_{t}\right\}
$$

is closed.

Let $x \in K_{0}$. Then $\pi(x)=1$. Consider the idempotent $e_{1}=e(1, x)$. By Lemma 2, $x e_{1}=e_{1}$. Hence $e e_{1}=e x e_{1}=x e_{1}=e_{1}$. In the same way, we obtain $e_{1} e=e_{1}$. On the other hand, by Lemma $1,1-e_{1} \in J$ and, by Lemma $2,\left\|e_{1}\right\|=1$. The minimality of $e$ implies $e_{1}=e$. Thus, it follows that $x=x e=x e_{1}=e_{1}=e$. In other words, $K_{0}$ is the singleton $\{e\}$. From the relation $K_{t} K_{-t} \subseteq K_{0}=\{e\}$ we can show that each $K_{t}$ is a singleton, say $K_{t}=\left\{x_{t}\right\}$. From the fact that the graph of $t \rightarrow K_{t}$ is closed it follows that $t \rightarrow x_{t}$ is continuous. Thus we obtain a one-parameter group $\left\{x_{t}\right\}$ of unitary elements in eAe with $\pi\left(x_{t}\right)=\exp (i t h)$. Let $\tilde{h}=\lim _{t \rightarrow 0}\left[\left(x_{t}-e\right) / i t\right]$. Then $\tilde{h}$ is a hermitian element in $e A e$ and $\pi(\tilde{h})=h$.

Proof of Theorem B. Let $x$ be an element in $A$ such that $\|x\|=1$ and $\pi(x)=u$. For $\lambda \in \operatorname{Sp}(x)$, we write $e_{\lambda}$ for the idempotent $e(\lambda, x)$. Since $u$ is unitary, $\operatorname{Sp}(u)$ is contained in the unit circle. Hence, by Lemma 1 , if $\lambda \in \operatorname{Sp}(x)$ and $|\lambda|<1$, then $e_{\lambda} \in J$. Let $F=$ $\operatorname{Sp}(x) \cap\{\lambda:|\lambda|=1\}$ and $e_{F}=\sum_{\lambda \in F} e_{\lambda}$. Then $1-e_{F} \in J$ and, by Lemma 2 ,

$$
x=\sum_{\lambda \in F} \lambda e_{\lambda}+z
$$

where $z=x\left(1-e_{F}\right) \in J$, has spectral radius less than 1 . Choose an increasing sequence $\left\{n_{k}\right\}$ of positive integers such that

(1) $m_{k}=n_{k+1}-n_{k} \rightarrow \infty$ as $k \rightarrow \infty$, and

(2) for all $\lambda \in F, \lambda^{n_{k}} \rightarrow \mu_{\lambda}$ for some $\mu_{\lambda}$ as $k \rightarrow \infty$. 
Then $x^{m_{k}+1}=\sum_{\lambda \in F} \lambda^{n_{k+1}-n_{k}} e_{\lambda}+z^{m_{k}}$ which tends to $e_{F}=\sum_{\lambda \in F} e_{\lambda}$ as $k \rightarrow \infty$. Since $\left\|x^{m_{k}}\right\| \leq 1$ for all $k$, we have $\left\|e_{F}\right\|=1$. Note that $x^{n_{k+1}}$ and $x^{n_{k-1}}$ tend to $\tilde{u}=\sum \lambda e_{\lambda}$ and $\tilde{v}=\sum \bar{\lambda} e_{\lambda}$ respectively. Hence we obtain $\|\tilde{u}\| \leq 1,\|\tilde{v}\| \leq 1$. Obviously $\tilde{u} \tilde{v}=\tilde{v} \tilde{u}=e_{F}$. Hence $\tilde{u}$ is a unitary element in $e_{F} A e_{F}$ with $1-e_{F} \in J$ and $\pi(\tilde{u})=u$. Here the conclusion is slightly different from the statement of the theorem. This can be adjusted by choosing $x$ at the beginning that satisfies the additional condition $x \in e A e$, from which we can deduce $e=e_{F}$. The proof is complete.

Remarks. In Theorem $A$, the assumption that $A$ is finite dimensional can be replaced by a weaker one that $J$ is finite dimensional with a modified proof.

2. From the proof of Theorem B it follows that if $A$ is a finite dimensional algebra and $x$ is an element in $A$ with its spectrum contained in the unit circle and $\|x\|=1$, then $x$ is unitary.

\section{REFERENCES}

1. G. D. Allen and J. D. Ward, Hermitian liftings in $B\left(\ell_{p}\right)$. To appear.

2. G. D. Allen, J. D. Ward and D. A. Legg, Hermitian liftings in Orlicz sequence spaces. To appear.

3. F. F. Bonsall and J. Duncan, Complete normed algebras. Ergebnisse der Mathematik and ihrer Grenzgebiete, Band 80. (Springer-Verlag, 1973).

4. A. M. Sinclair, Eigenvalues in the boundary of the numerical range, Pacific J. Math. 35 (1970), 231-234.

Department of Mathematics and Statistics,

UNIVERISTY OF GUELPH,

Guelph, Ontario, Canada. 